\title{
Inequalities between the inverse hyperbolic tangent and the inverse sine and the analogue for corresponding functions
}

Ling Zhu ${ }^{1 *}$ and Branko Malešević ${ }^{2}$

\section{"Correspondence:}

zhuling0571@163.com

${ }^{1}$ Department of Mathematics,

Zhejiang Gongshang University,

Hangzhou, China

Full list of author information is

available at the end of the article

\begin{abstract}
In this paper, we obtain some new inequalities which reveal the further relationship between the inverse tangent function $\arctan x$ and the inverse hyperbolic sine function $\sinh ^{-1} x$. At the same time, we give the analogue for inverse hyperbolic tangent and inverse sine.
\end{abstract}

MSC: Primary 26D05; 26D15; secondary 33B10

Keywords: Inequalities; Inverse tangent function; Inverse hyperbolic sine function; Inverse hyperbolic tangent function; Inverse sine function

\section{Introduction}

In 2010, Masjed-Jamei [1] obtained the following inequality:

$$
(\arctan x)^{2} \leq \frac{x \ln \left(x+\sqrt{1+x^{2}}\right)}{\sqrt{1+x^{2}}}, \quad|x|<1
$$

[1] also reminded us that the above inequality is established in a larger interval $(-\infty, \infty)$ because it was detected by Maple software. Inequality (1.1) gives the upper bound for the square of the inverse tangent function $\arctan x$ by the inverse hyperbolic sine function $\sinh ^{-1} x=\ln \left(x+\sqrt{1+x^{2}}\right)$.

In this paper, we first affirm Masjed-Jamei's quest, conclude that the scope of the inequality is indeed the large interval $(-\infty, \infty)$, and give a simple proof of this result. Second, we get the strengthening of the inequality that we have just given. Then, we obtain some natural generalizations of this inequality. At the same time, we show the analogue for inverse hyperbolic tangent function $\operatorname{arctanh} x=(1 / 2) \ln ((1+x) /(1-x))$ and inverse sine function $\arcsin x$. Finally, we propose a conjecture on this topic.

Theorem 1.1 The inequality

$$
(\arctan x)^{2} \leq \frac{x \ln \left(x+\sqrt{1+x^{2}}\right)}{\sqrt{1+x^{2}}}
$$

holds for all $x \in(-\infty, \infty)$, and the power number 2 is the best in (1.2).

(c) The Author(s) 2019. This article is distributed under the terms of the Creative Commons Attribution 4.0 International License (http://creativecommons.org/licenses/by/4.0/), which permits unrestricted use, distribution, and reproduction in any medium, provided you give appropriate credit to the original author(s) and the source, provide a link to the Creative Commons license, and indicate if changes were made. 
Theorem 1.2 Let $0<r<\infty, \lambda=1$, and $\mu=r \ln \left(r+\sqrt{r^{2}+1}\right) /\left(\sqrt{r^{2}+1}(\arctan r)^{2}\right)$. Then the double inequality

$$
\lambda(\arctan x)^{2} \leq \frac{x \ln \left(x+\sqrt{1+x^{2}}\right)}{\sqrt{1+x^{2}}} \leq \mu(\arctan x)^{2}
$$

holds for all $x \in(-r, r)$, where $\lambda$ and $\mu$ are the best constants in (1.3).

Theorem 1.3 Let $-\infty<x<\infty$. Then we have

$$
\begin{aligned}
-\frac{1}{45} x^{6} \leq(\arctan x)^{2}-\frac{x \ln \left(x+\sqrt{1+x^{2}}\right)}{\sqrt{1+x^{2}}} & \leq-\frac{1}{45} x^{6}+\frac{4}{105} x^{8}, \\
-\frac{1}{45} x^{6}+\frac{4}{105} x^{8}-\frac{11}{225} x^{10} & \leq(\arctan x)^{2}-\frac{x \ln \left(x+\sqrt{1+x^{2}}\right)}{\sqrt{1+x^{2}}} \\
& \leq-\frac{1}{45} x^{6}+\frac{4}{105} x^{8}-\frac{11}{225} x^{10}+\frac{586}{10,395} x^{12} .
\end{aligned}
$$

Theorem 1.4 The inequality

$$
(\operatorname{arctanh} x)^{2} \leq \frac{x \arcsin x}{\sqrt{1-x^{2}}}
$$

holds for all $x \in(-1,1)$, and the power number 2 is the best in (1.6).

Theorem 1.5 Let $0<r<1, \alpha=1$, and $\beta=r(\arcsin r) /\left(\sqrt{1-r^{2}}(\operatorname{arctanh} r)^{2}\right)$. Then the double inequality

$$
\alpha(\operatorname{arctanh} x)^{2} \leq \frac{x \arcsin x}{\sqrt{1-x^{2}}} \leq \beta(\operatorname{arctanh} x)^{2}
$$

holds for all $x \in(-r, r)$, where $\alpha$ and $\beta$ are the best constants in (1.7).

Theorem 1.6 Let $n, N$ be two integers, $n, N \geq 3$, and

$$
v_{n}=\frac{1}{n}\left(\frac{n ! 2^{n-1}}{(2 n-1) ! !}-\left(1+\frac{1}{3}+\cdots+\frac{1}{2 n-1}\right)\right) .
$$

Then the inequality

$$
\frac{x \arcsin x}{\sqrt{1-x^{2}}}-(\operatorname{arctanh} x)^{2} \geq \sum_{n=3}^{N} v_{n} x^{2 n}
$$

holds for all $x \in(-1,1)$.

\section{Simple proof of Theorem 1.1}

Let $\arctan x=t, x \in(-\infty, \infty)$. Then $x=\tan t, t \in(-\pi / 2, \pi / 2)$, and (1.2) is equivalent to

$$
\ln (\tan t+\sec t)=\ln \frac{1+\sin t}{\cos t}>\frac{t^{2}}{\sin t}
$$


for $t \neq 0$ since the equality in (1.2) holds for $x=0$. Let

$$
F_{1}(t)=\ln \frac{1+\sin t}{\cos t}-\frac{t^{2}}{\sin t}=\ln (1+\sin t)-\ln \cos t-\frac{t^{2}}{\sin t} .
$$

Then

$$
\begin{aligned}
F_{1}^{\prime}(t) & =\frac{\cos t}{\sin t+1}+\frac{1}{\cos t} \sin t+\frac{1}{\sin ^{2} t}\left(t^{2} \cos t-2 t \sin t\right) \\
& =\frac{1}{\cos t}+\frac{1}{\sin ^{2} t}\left(t^{2} \cos t-2 t \sin t\right)=\frac{(-\sin t+t \cos t)^{2}}{\cos t \sin ^{2} t},
\end{aligned}
$$

which means that $F_{1}^{\prime}(t)>0$ for all $t \in(0, \pi / 2)$ and $F_{1}^{\prime}(t)<0$ for all $t \in(-\pi / 2,0)$. So $F_{1}(t)>$ $F_{1}\left(0^{+}\right)=0$ for all $t \in(-\pi / 2,0) \cup(0, \pi / 2)$. In view of

$$
\lim _{x \rightarrow 0} \frac{\ln \frac{x \ln \left(x+\sqrt{1+x^{2}}\right)}{\sqrt{1+x^{2}}}}{\ln \tan ^{-1} x}=2,
$$

the proof of Theorem 1.1 is complete.

\section{Proof of Theorem 1.2}

In order to prove Theorem 1.2, we use a key method as follows, which is called the monotone form of l'Hospital's rule.

Lemma $3.1([2,3])$ For $-\infty<a<b<\infty$, let $f, g:[a, b] \rightarrow \mathbb{R}$ be continuous functions that are differentiable on $(a, b)$, with $f(a)=g(a)=0$ or $f(b)=g(b)=0$. If $f^{\prime} / g^{\prime}$ with $g^{\prime}(x) \neq 0$ for each $x$ in $(a, b)$ is increasing (decreasing) on $(a, b)$, then so is $f / g$.

Now, we are in the state of proving Theorem 1.2. After making the same transformation with the second section, we obtain that $t \in(-\arctan r, \arctan r) \subset(-\pi / 2, \pi / 2)$. Considering that the two functions involved in (1.3) are even functions, we can discuss problems in the range $(0, \arctan r)$. Let

$$
\begin{aligned}
G_{1}(t) & =\frac{t^{2}}{\frac{(\tan t) \ln (\tan t+\sec t)}{\sec t}}=\frac{t^{2}}{(\sin t)(\ln (1+\sin t)-\ln \cos t)} \\
& =\frac{\frac{t^{2}}{\sin t}}{\ln (1+\sin t)-\ln \cos t}:=\frac{f_{1}(t)}{g_{1}(t)}
\end{aligned}
$$

where

$$
f_{1}(t)=\frac{t^{2}}{\sin t}, \quad g_{1}(t)=\ln (1+\sin t)-\ln \cos t
$$

Then

$$
f_{1}^{\prime}(t)=\frac{2 t \sin t-t^{2} \cos t}{\sin ^{2} t}, \quad g_{1}^{\prime}(t)=\frac{1}{\cos t}
$$

and

$$
\frac{f_{1}^{\prime}(t)}{g_{1}^{\prime}(t)}=2 t \cot t-t^{2} \cot ^{2} t
$$


Since

$$
\begin{aligned}
\left(\frac{f_{1}^{\prime}(t)}{g_{1}^{\prime}(t)}\right)^{\prime} & =2 t^{2} \cot ^{3} t+2 t^{2} \cot t-4 t \cot ^{2} t-2 t+2 \cot t \\
& =2(t \cot t-1)\left(t-\cot t+t \cot ^{2} t\right) \\
& =2(t \cot t-1)\left(\frac{t}{\sin ^{2} t}-\frac{\cos t}{\sin t}\right) \\
& =2(t \cot t-1) \frac{t-\sin t \cos t}{\sin ^{2} t}<0
\end{aligned}
$$

we have that the function $f_{1}^{\prime}(t) / g_{1}^{\prime}(t)$ is decreasing on $(0, \arctan r)$. Then $G_{1}(t)=f_{1}(t) / g_{1}(t)$ is decreasing on $(0, \arctan r)$ too by Lemma 3.1. In view of

$$
\begin{aligned}
& \frac{1}{\lambda}:=\lim _{t \rightarrow 0^{+}} G_{1}(t)=1, \\
& \frac{1}{\mu}:=\lim _{t \rightarrow \arctan r} G_{1}(t)=\frac{\sqrt{r^{2}+1}(\arctan r)^{2}}{r \ln \left(r+\sqrt{r^{2}+1}\right)},
\end{aligned}
$$

the proof of Theorem 1.2 is complete.

Remark 3.1 Letting $r \rightarrow \infty$ in Theorem 1.2, we can obtain Theorem 1.1.

\section{Proof of Theorem 1.3}

Because the functions involved in this section are all even functions, we only assume $x>0$. After doing the same transformation with the second section, we will only discuss problems in the situation $t \in(0, \pi / 2)$. Let

$$
\begin{aligned}
& h_{1}(t)=\frac{t^{2}-(\sin t) \ln \frac{1+\sin t}{\cos t}+\frac{1}{45} \tan ^{6} t}{\sin t}=\frac{t^{2}}{\sin t}-\ln \frac{1+\sin t}{\cos t}+\frac{1}{45} \frac{\tan ^{6} t}{\sin t}, \\
& h_{2}(t)=\frac{t^{2}}{\sin t}-\ln \frac{1+\sin t}{\cos t}+\frac{1}{45} \frac{\tan ^{6} t}{\sin t}-\frac{4}{105} \frac{\tan ^{8} t}{\sin t}, \\
& h_{3}(t)=\frac{t^{2}}{\sin t}-\ln \frac{1+\sin t}{\cos t}+\frac{1}{45} \frac{\tan ^{6} t}{\sin ^{2}}-\frac{4}{105} \frac{\tan ^{8} t}{\sin t}+\frac{11}{225} \frac{\tan ^{10} t}{\sin ^{10}}, \\
& h_{4}(t)=\frac{t^{2}}{\sin t}-\ln \frac{1+\sin t}{\cos t}+\frac{1}{45} \frac{\tan ^{6} t}{\sin t}-\frac{4}{105} \frac{\tan ^{8} t}{\sin t}+\frac{11}{225} \frac{\tan ^{10} t}{\sin t}-\frac{586}{10,395} \frac{\tan ^{12} t}{\sin t} .
\end{aligned}
$$

Then we get $h_{i}\left(0^{+}\right)=0, i=1,2,3,4$, and

$$
\begin{aligned}
& h_{1}^{\prime}(t)=\frac{\cos t}{45 \sin ^{2} t} g_{1}(t), \\
& h_{2}^{\prime}(t)=-\frac{\cos t}{315 \sin ^{2} t} g_{2}(t), \\
& h_{3}^{\prime}(t)=\frac{\cos t}{1575 \sin ^{2} t} g_{3}(t), \\
& h_{4}^{\prime}(t)=-\frac{\cos t}{51,975 \sin ^{2} t} g_{4}(t),
\end{aligned}
$$


where

$$
\begin{aligned}
g_{1}(t)= & -45 t^{2}+90 t \tan t-45 \tan ^{2} t+5 \tan ^{6} t+6 \tan ^{8} t, \\
g_{2}(t)= & 315 t^{2}-630 t \tan t+315 \tan ^{2} t-35 \tan ^{6} t+42 \tan ^{8} t+96 \tan ^{10} t, \\
g_{3}(t)= & -1575 t^{2}+3150 t \tan t-1575 \tan ^{2} t+175 \tan ^{6} t-210 \tan ^{8} t \\
& +213 \tan ^{10} t+770 \tan ^{12} t, \\
g_{4}(t)= & 51,975 t^{2}-103,950 t \tan t+51,975 \tan ^{2} t-5775 \tan ^{6} t+6930 \tan ^{8} t \\
& -7029 \tan ^{10} t+6820 \tan ^{12} t+35,160 \tan ^{14} t,
\end{aligned}
$$

and $g_{i}(0)=0, i=1,2,3,4$. We compute to get

$$
\begin{aligned}
f_{1}(t) & :=\frac{g_{1}^{\prime}(t)}{6 \tan ^{2} t}=8 \tan ^{7} t+13 \tan ^{5} t+5 \tan ^{3} t-15 \tan t+15 t, \\
f_{2}(t):= & \frac{g_{2}^{\prime}(t)}{6 \tan ^{2} t}=160 \tan ^{9} t+216 \tan ^{7} t+21 \tan ^{5} t-35 \tan ^{3} t+105 \tan t-105 t, \\
f_{3}(t):= & \frac{g_{3}^{\prime}(t)}{30 \tan ^{2} t} \\
= & 308 \tan ^{11} t+379 \tan ^{9} t+15 \tan ^{7} t-21 \tan ^{5} t+35 \tan ^{3} t-105 \tan t+105 t, \\
f_{4}(t):= & \frac{g_{3}^{\prime}(t)}{30 \tan ^{2} t}=16,408 \tan ^{13} t+19,136 \tan ^{11} t+385 \tan ^{9} t-495 \tan ^{7} t+693 \tan ^{5} t \\
& -1155 \tan ^{3} t+3465 \tan t-3465 t
\end{aligned}
$$

with $f_{i}(0)=0, i=1,2,3,4$. Then

$$
\begin{aligned}
& f_{1}^{\prime}(t)=\left(\tan ^{4} t\right)\left(56 \tan ^{4} t+121 \tan ^{2} t+80\right)>0, \\
& f_{2}^{\prime}(t)=\left(\tan ^{6} t\right)\left(1440 \tan ^{4} t+2952 \tan ^{2} t+1617\right)>0, \\
& f_{3}^{\prime}(t)=\left(\tan ^{8} t\right)\left(3388 \tan ^{4} t+6799 \tan ^{2} t+3516\right)>0, \\
& f_{4}^{\prime}(t)=\left(\tan ^{10} t\right)\left(213,304 \tan ^{4} t+423,800 \tan ^{2} t+213,961\right)>0 .
\end{aligned}
$$

Through differential deduction, we complete the proof of Theorem 1.3.

\section{Proof of Theorem 1.4}

Since the two functions showed in (1.6) are even functions, we can discuss problems in the range $(0,1)$. Let $\arcsin x=t, x \in(0,1)$. Then $x=\sin t, t \in(0, \pi / 2)$. We find that

$$
\begin{aligned}
\operatorname{arctanh}(\sin t) & =\frac{1}{2} \ln \frac{1+\sin t}{1-\sin t}=\frac{1}{2} \ln \frac{(1+\sin t)^{2}}{(1-\sin t)(1+\sin t)} \\
& =\frac{1}{2} \ln \left(\frac{1+\sin t}{\cos t}\right)^{2}=\ln \frac{1+\sin t}{\cos t},
\end{aligned}
$$

and (1.6) is equivalent to

$$
\left(\ln \frac{1+\sin t}{\cos t}\right)^{2}<\frac{t \sin t}{\cos t}, \quad 0<t<\frac{\pi}{2}
$$


Let

$$
F_{2}(t)=\frac{t \sin t}{\cos t}-\left(\ln \frac{1+\sin t}{\cos t}\right)^{2} .
$$

Then

$$
F_{2}^{\prime}(t)=\frac{t+\cos t \sin t}{\cos ^{2} t}-\frac{2}{\cos t} \ln \frac{\sin t+1}{\cos t},
$$

or

$$
(\cos t) F_{2}^{\prime}(t)=\frac{t+\cos t \sin t}{\cos t}-2 \ln \frac{\sin t+1}{\cos t} .
$$

We can compute to obtain

$$
\left((\cos t) F_{2}^{\prime}(t)\right)^{\prime}=\frac{(\sin t)(2 t-\sin 2 t)}{2 \cos ^{2} t}>0, \quad 0<t<\frac{\pi}{2},
$$

which implies that

$$
(\cos t) F_{2}^{\prime}(t)>\lim _{t \rightarrow 0^{+}}(\cos t) F_{2}^{\prime}(t)=0
$$

for all $t \in(0, \pi / 2)$. Then

$$
F_{2}^{\prime}(t)>0 \quad \Longrightarrow \quad F_{2}(t)>F_{2}\left(0^{+}\right)=0
$$

for all $t \in(0, \pi / 2)$.

In view of

$$
\lim _{x \rightarrow 0} \frac{\ln \frac{x \arcsin x}{\sqrt{1-x^{2}}}}{\ln \operatorname{arctanh} x}=2,
$$

the proof of Theorem 1.4 is complete.

\section{Proof of Theorem 1.5}

After making the same transformation as in the section above, we obtain that $t \in$ $(-\arcsin r, \arcsin r) \subset(-\pi / 2, \pi / 2)$. Considering that the two functions involved in (1.7) are even functions, we can discuss problems in the range $(0, \arcsin r)$. Let

$$
G_{2}(t)=\frac{\frac{t(\sin t)}{\cos t}}{\left(\ln \frac{1+\sin t}{\cos t}\right)^{2}}:=\frac{f_{2}(t)}{g_{2}(t)},
$$

where

$$
f_{2}(t)=\frac{t(\sin t)}{\cos t}, \quad g_{2}(t)=\left(\ln \frac{1+\sin t}{\cos t}\right)^{2} .
$$

Then

$$
f_{2}^{\prime}(t)=\frac{t+\cos t \sin t}{\cos ^{2} t}, \quad g_{2}^{\prime}(t)=\frac{2}{\cos t} \ln \frac{\sin t+1}{\cos t}
$$


and

$$
\frac{f_{2}^{\prime}(t)}{g_{2}^{\prime}(t)}=\frac{\frac{t+\cos t \sin t}{\cos ^{2} t}}{\frac{2}{\cos t} \ln \frac{\sin t+1}{\cos t}}=\frac{\frac{t+\cos t \sin t}{\cos t}}{2 \ln \frac{\sin t+1}{\cos t}}:=\frac{f_{3}(t)}{g_{3}(t)} .
$$

Since

$$
\begin{aligned}
& f_{3}^{\prime}(t)=\left(\frac{t+\cos t \sin t}{\cos t}\right)^{\prime}=\frac{1}{\cos ^{2} t}\left(\cos ^{3} t+\cos t+t \sin t\right) \\
& g_{3}^{\prime}(t)=\left(2 \ln \frac{\sin t+1}{\cos t}\right)^{\prime}=\frac{2}{\cos t},
\end{aligned}
$$

we have

$$
\frac{f_{3}^{\prime}(t)}{g_{3}^{\prime}(t)}=\frac{1}{2 \cos t}\left(\cos ^{3} t+\cos t+t \sin t\right) .
$$

So

$$
\left(\frac{f_{3}^{\prime}(t)}{g_{3}^{\prime}(t)}\right)^{\prime}=\frac{1}{8 \cos ^{2} t}(4 t-\sin 4 t)>0
$$

which leads to the fact that the function $f_{3}^{\prime}(t) / g_{3}^{\prime}(t)$ is increasing on $(0, \arcsin r)$. Then $f_{3}(t) / g_{3}(t)$ is increasing on $(0, \arcsin r)$ too by Lemma 3.1. Using Lemma 3.1 again, we come to the conclusion that $G_{2}(t)=f_{2}(t) / g_{2}(t)$ is increasing on $(0, \arcsin r)$.

In view of

$$
\begin{aligned}
& \alpha:=\lim _{t \rightarrow 0^{+}} G_{2}(t)=1, \\
& \beta:=\lim _{t \rightarrow \arcsin r} G_{2}(t)=\frac{r \arcsin r}{(\operatorname{arctanh} r)^{2} \sqrt{1-r^{2}}},
\end{aligned}
$$

the proof of Theorem 1.5 is complete.

Remark 6.1 Letting $r \rightarrow 1$ in Theorem 1.5, we can obtain Theorem 1.4.

\section{Proof of Theorem 1.6}

In order to prove Theorem 1.6, we need the following lemma.

Lemma $7.1([4-8])$ Let $|x|<1$. Then

$$
\frac{2 x \arcsin x}{\sqrt{1-x^{2}}}=\sum_{n=1}^{\infty} \frac{(2 x)^{2 n}}{n\left(\begin{array}{c}
2 n \\
n
\end{array}\right)} .
$$

We are in the state of proving Theorem 1.6.

First, by Lemma 7.1 we get

$$
\frac{x \arcsin x}{\sqrt{1-x^{2}}}=\sum_{n=1}^{\infty} \frac{n ! 2^{n-1}}{n(2 n-1) ! !} x^{2 n}
$$


due to

$$
\left(\begin{array}{c}
2 n \\
n
\end{array}\right)=\frac{2^{n}(2 n-1) ! !}{n !}
$$

Second, we have

$$
\begin{aligned}
\frac{d}{d x}(\operatorname{arctanh} x)^{2} & =\frac{2}{1-x^{2}} \operatorname{arctanh} x=2\left(\sum_{n=0}^{\infty} x^{2 n}\right)\left(\sum_{n=0}^{\infty} \frac{x^{2 n+1}}{2 n+1}\right) \\
& =2 \sum_{n=1}^{\infty}\left(1+\frac{1}{3}+\cdots+\frac{1}{2 n-1}\right) x^{2 n-1} .
\end{aligned}
$$

Integrating two sides of (7.3) on $[0, x]$, we can obtain

$$
(\operatorname{arctanh} x)^{2}=\sum_{n=1}^{\infty} \frac{1}{n}\left(1+\frac{1}{3}+\cdots+\frac{1}{2 n-1}\right) x^{2 n}
$$

From (7.2) and (7.4) we have

$$
\begin{aligned}
\frac{x \arcsin x}{\sqrt{1-x^{2}}}-(\operatorname{arctanh} x)^{2} & =\sum_{n=1}^{\infty}\left(\frac{n ! 2^{n-1}}{n(2 n-1) ! !} x^{2 n}-\frac{1}{n}\left(1+\frac{1}{3}+\cdots+\frac{1}{2 n-1}\right)\right) x^{2 n} \\
& =\sum_{n=3}^{\infty} \frac{1}{n}\left(\frac{n ! 2^{n-1}}{(2 n-1) ! !} x^{2 n}-\left(1+\frac{1}{3}+\cdots+\frac{1}{2 n-1}\right)\right) x^{2 n} \\
& :=\sum_{n=3}^{\infty} v_{n} x^{2 n}
\end{aligned}
$$

where

$$
v_{n}=\frac{1}{n}\left(\frac{n ! 2^{n-1}}{(2 n-1) ! !}-\left(1+\frac{1}{3}+\cdots+\frac{1}{2 n-1}\right)\right), \quad n \geq 3 .
$$

Below we shall prove that

$$
u_{n}:=n v_{n}=\frac{n ! 2^{n-1}}{(2 n-1) ! !}-\left(1+\frac{1}{3}+\cdots+\frac{1}{2 n-1}\right)>0
$$

for $n \geq 3$.

In fact, when $n=3$, inequality (7.6) holds. Now, we assume that (7.6) holds for $n=m$, that is,

$$
\frac{m ! 2^{m-1}}{(2 m-1) ! !}>1+\frac{1}{3}+\cdots+\frac{1}{2 m-1}
$$

Since

$$
\frac{(m+1) ! 2^{m}}{(2 m+1) ! !}=\frac{2(m+1)}{2 m+1} \frac{m ! 2^{m-1}}{(2 m-1) ! !}>\frac{2(m+1)}{2 m+1}\left(1+\frac{1}{3}+\cdots+\frac{1}{2 m-1}\right)
$$


in order to prove that (7.6) is also true for $n=m+1$, it suffices to show that

$$
\frac{2(m+1)}{2 m+1}\left(1+\frac{1}{3}+\cdots+\frac{1}{2 m-1}\right)>\left(1+\frac{1}{3}+\cdots+\frac{1}{2 m-1}\right)+\frac{1}{2 m+1},
$$

which is true due to

$$
\frac{1}{2 m+1}\left(1+\frac{1}{3}+\cdots+\frac{1}{2 m-1}\right)>\frac{1}{2 m+1}
$$

or

$$
1+\frac{1}{3}+\cdots+\frac{1}{2 m-1}>1
$$

So, $v_{n}>0$ for $n \geq 3$, and

$$
\frac{x \arcsin x}{\sqrt{1-x^{2}}}-(\operatorname{arctanh} x)^{2} \geq \sum_{n=3}^{N} v_{n} x^{2 n}
$$

holds for all $x \in(-1,1)$, where $N$ is any integer greater than or equal to 3 .

Remark 7.1 Theorem 1.6 is obviously a natural extension of Theorem 1.4.

\section{Conjecture}

Inspired by [9], in the last section, we pose the following conjecture in the form of (1.4) and (1.5).

Conjecture 8.1 Let $x \in \mathbf{R}, m \geq 1$, and $v_{n}$ as defined by (1.8). Then the double inequality

$$
\sum_{n=3}^{2 m+1}(-1)^{n} v_{n} x^{2 n} \leq(\arctan x)^{2}-\frac{x \ln \left(x+\sqrt{1+x^{2}}\right)}{\sqrt{1+x^{2}}} \leq \sum_{n=3}^{2 m+2}(-1)^{n} v_{n} x^{2 n}
$$

holds.

Remark 8.1 There are several factors that lead to the fact that this double inequality cannot be proved by Leibniz's theorem for alternating series. The first is that the interval we are discussing now is infinite, and the second is that the sequence $\left\{v_{n}\right\}_{n \geq 3}$ does not have the characteristic of monotone decreasing.

Funding

The first author was supported by the National Natural Science Foundation of China (no. 11471285 and no. 61772025). The second author was supported in part by the Serbian Ministry of Education, Science and Technological Development, under projects ON 174032 and III 44006.

Competing interests

The authors declare that they have no competing interests.

Authors' contributions

The authors provided the questions and gave the proof for all the results. They read and approved this manuscript.

\section{Author details}

${ }^{1}$ Department of Mathematics, Zhejiang Gongshang University, Hangzhou, China. ${ }^{2}$ Faculty of Electrical Engineering, University of Belgrade, Belgrade, Serbia. 


\section{Publisher's Note}

Springer Nature remains neutral with regard to jurisdictional claims in published maps and institutional affiliations.

Received: 7 January 2019 Accepted: 28 March 2019 Published online: 03 April 2019

\section{References}

1. Masjed-Jamei, M.: A main inequality for several special functions. Comput. Math. Appl. 60, 1280-1289 (2010)

2. Anderson, G.D., Vamanamurthy, M.K., Vuorinen, M.: Conformal Invariants, Inequalities, and Quasiconformal Maps, Wiley, New York (1997)

3. Anderson, G.D., Qiu, S.L., Vamanamurthy, M.K., Vuorinen, M.: Generalized elliptic integral and modular equations. Pac. J. Math. 192, 1-37 (2000)

4. Lehmer, D.H.: Interesting series involving the central binomial coefficient. Am. Math. Mon. 92, 449-457 (1985)

5. Sury, B., Wang, T.M., Zhao, F.Z.: Identities involving reciprocals of binomial coefficients. J. Integer Seq. 7, Article 04.2.8 (2004)

6. Chen, H.W.: A power series expansion and its applications. Int. J. Math. Educ. Sci. Technol. 37(3), 362-368 (2006)

7. Sofo, A.: Integral identities involving reciprocals of single and double binomial coefficients. RGMIA Seminar Series (2007)

8. Borwein, J.M., Chamberland, M.: Integer powers of arcsin. Int. J. Math. Math. Sci. 2007, Article ID 19381 (2007). https://doi.org/10.1155/2007/19381

9. Malešević, B., Lutovac, T., Rašajski, M., Mortici, C.: Extensions of the natural approach to refinements and generalizations of some trigonometric inequalities. Adv. Differ. Equ. 2018, 90 (2018). https://doi.org/10.1186/s13662-018-1545-7

\section{Submit your manuscript to a SpringerOpen ${ }^{\circ}$ journal and benefit from:}

- Convenient online submission

- Rigorous peer review

- Open access: articles freely available online

- High visibility within the field

Retaining the copyright to your article 